\title{
BMJ
}

\section{Radial extracorporeal shockwave treatment compared with supervised exercises in patients with subacromial pain syndrome: single blind randomised study}

\author{
Kaia Engebretsen, physiotherapist, ${ }^{1,2}$ Margreth Grotle, research leader, ${ }^{2,3}$ Erik Bautz-Holter, professor, ${ }^{1,2}$ Leiv \\ Sandvik, professor, ${ }^{4}$ Niels G Juel, MD consultant, ${ }^{1,2}$ Ole Marius Ekeberg, Research fellow, ${ }^{1,2}$ Jens Ivar Brox, MD \\ consultant 2,5
}

${ }^{1}$ Department of Physical Medicine and Rehabilitation, Ullevaal

University Hospital, Kirkeveien 166, 0407 Oslo, Norway

${ }^{2}$ Medical Faculty, University of Oslo, Oslo

${ }^{3}$ FORMI, Division for Neuroscience and Musculoskeletal Medicine,

Ullevaal University Hospital

${ }^{4}$ Section for Biostatistics and Epidemiology, Ullevaal University Hospital

${ }^{5}$ Department of Orthopaedics, Section for Physical Medicine and Rehabilitation, National Hospital, Oslo

Correspondence to: $\mathrm{K}$

Engebretsen

kaiabe@medisin.uio.no

Cite this as: BMJ 2009;339:b3360 doi:10.1136/bmj.b3360

\section{ABSTRACT}

Objective To compare the effectiveness of radial extracorporeal shockwave treatment with that of supervised exercises in patients with shoulder pain. Design Single blind randomised study.

Setting Outpatient clinic of physical medicine and rehabilitation department in Oslo, Norway.

Participants 104 patients with subacromial shoulder pain lasting at least three months.

Interventions Radial extracorporeal shockwave treatment: one session weekly for four to six weeks. Supervised exercises: two 45 minute sessions weekly for up to 12 weeks.

Primary outcome measure Shoulder pain and disability index.

Results A treatment effect in favour of supervised exercises at 6,12 , and 18 weeks was found. The adjusted treatment effect was -8.4 (95\% confidence interval -16.5 to -0.6$)$ points. A significantly higher proportion of patients in the group treated with supervised exercises improved-odds ratio 3.2 (1.3 to 7.8). More patients in the shockwave treatment group had additional treatment between 12 and 18 weeks-odds ratio 5.5 (1.3 to 26.4). Conclusion Supervised exercises were more effective than radial extracorporeal shockwave treatment for short term improvement in patients with subacromial shoulder pain.

Trial registration Clinical trials NCT00653081.

\section{INTRODUCTION}

The shoulder is the fourth most common site of musculoskeletal pain reported by patients to general practitioners and physiotherapists. ${ }^{1}$ Rotator cuff disease, impingement syndrome, and rotator cuff tendinosis are terms used synonymously with subacromial shoulder pain. The exact source and mechanism of the pain is unknown. ${ }^{2}$ Histopathology studies show mainly degenerative changes in the rotator cuff tendons, but other factors also contribute to the pain and dysfunction. ${ }^{13}$

Patients with subacromial shoulder pain are often treated with physiotherapy, non-steroidal anti- inflammatory drugs, and corticosteroid injections. Physiotherapy includes a variety of modalities such as electrotherapy, radial extracorporeal shockwave treatment, ultrasound treatment, laser treatment, manual therapy, supervised exercises, sling exercise treatment, and acupuncture. ${ }^{4-7}$ Some evidence exists for the effectiveness of corticosteroid injections, non-steroidal antiinflammatory drugs, and exercises for chronic shoulder pain. ${ }^{8-10} \mathrm{~A}$ recent systematic review concluded that surgery and exercises are equally effective for rotator cuff disease. ${ }^{11}$ In studies of musculoskeletal pain, women report pain that is more severe, more frequent, and of a longer duration than do men. ${ }^{12}{ }^{13}$ Stratification by sex or adjustment for sex is therefore recommended in the analysis of data from trials.

In a systematic review, Harniman et al found moderate evidence that low energy radial extracorporeal shockwave treatment was not effective for non-calcifying rotator cuff tendinosis. ${ }^{6}$ Additional studies including patients with calcifying or non-calcifying tendinosis reported no treatment effect compared with sham or control. ${ }^{1415}$ Despite these findings, shockwave treatment is increasingly used for subacromial shoulder pain. To our knowledge, radial extracorporeal shockwave treatment has not been compared with exercises in a clinical trial including patients with shoulder pain. The purpose of this study was to compare the short term effect of radial extracorporeal shockwave treatment and supervised exercises in patients with subacromial shoulder pain.

\section{METHODS}

\section{Participants}

The study was designed as a randomised single blind clinical study. Participants were recruited by physicians at the outpatient clinic of the Physical Medicine and Rehabilitation Department at Ullevaal University Hospital, Oslo, Norway, between July 2006 and August 2007.

Women and men aged between 18 and 70 years with subacromial shoulder pain lasting at least three months were eligible for inclusion. The following diagnostic 


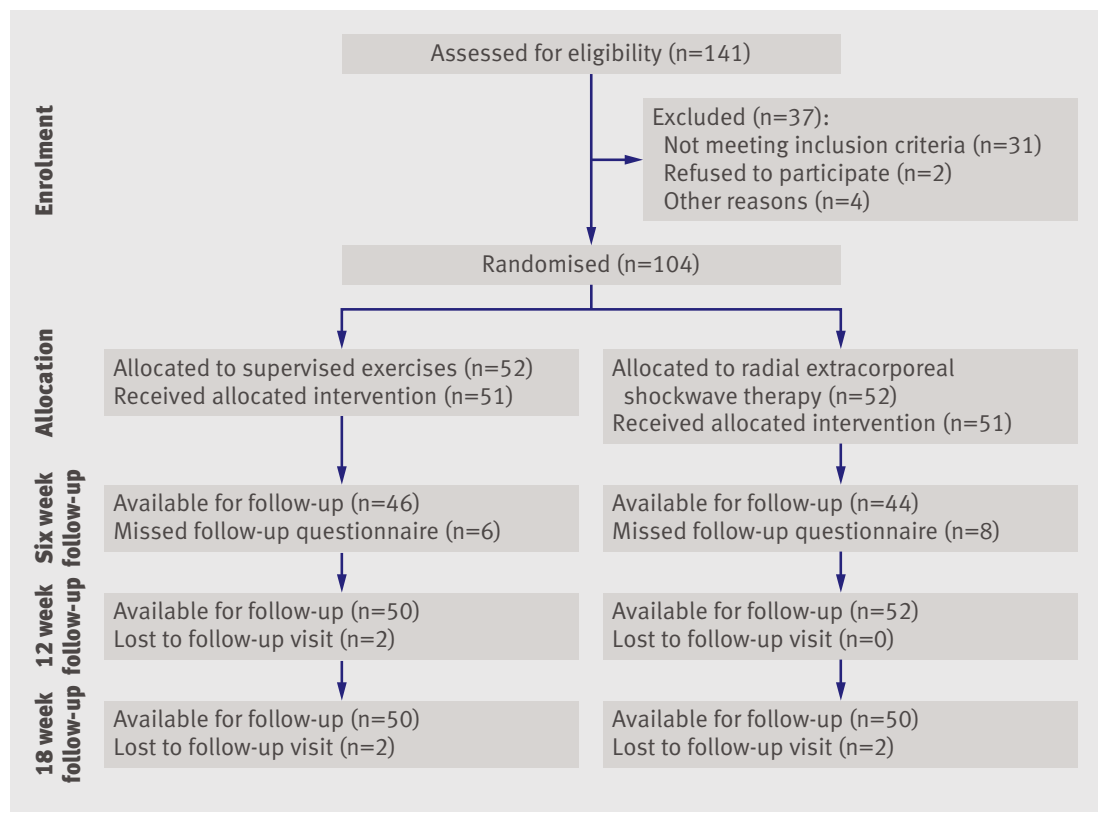

Fig 1| Flow chart of study outcome measure was the shoulder pain and disability index (SPADI), a self report questionnaire for patients with shoulder pain. The questionnaire consists of 13 questions divided into two domains: pain (five items) and disability (eight items). ${ }^{18}$ Answers are scored on visual analogue scales from $0 \mathrm{~cm}$ (best) to $11 \mathrm{~cm}$ (worst) and in accordance with the original scoring system. ${ }^{18}$ The total score ranges from 0 to 100 points, with a higher score indicating worse shoulder pain and disability. ${ }^{18}$ A version adapted to Norwegian language and culture, translated and back translated by independent philologists, was used in this study. ${ }^{1920}$

Intensity of pain during rest and activity in the previous week was measured on a nine point Lickert-type scale, on which 1 indicates no pain and 9 indicates severe pain. ${ }^{21}$ Participants were asked two questions about specific function of the shoulder: "Can you carry a shopping bag $(5 \mathrm{~kg})$ ?" and "Can you take down something from a wall cupboard?" These questions were scored on a scale from 1 (easy) to 7 (impossible)..$^{21}$ Active range of motion was measured bilaterally with an accuracy of $5^{\circ} .^{2122}$ The patients decided when the pain condition confined the movement.

Participants reported work status according to a validated questionnaire. ${ }^{23} \mathrm{~A}$ status of retired was recorded separately. They also recorded use of drug treatment, including both daily and weekly treatments for pain, sleep problems, and depression.

\section{Follow-up}

At six weeks the patients completed a postal questionnaire, including the outcome measures, at home. The 12 week and 18 week follow-ups were done at the hospital. A blinded physiotherapist made the baseline and follow-up measurements. The patients were instructed not to discuss their treatment with the blinded physiotherapist.

\section{Interventions}

Both treatments were given at the Department of Physical Medicine and Rehabilitation. The supervised exercise regimen, developed at Ullevaal University Hospital by Bøhmer in the $1980 \mathrm{~s},{ }^{5}$ was provided by two physiotherapists experienced in its performance. Patients attended two 45 minute sessions weekly for a maximum of 12 weeks. The first session included gathering medical history and bilateral inspection of alignment, including the scapula and the glenohumeral joint. Movement pattern, the immediate co-contraction, and timing of the scapula and the arm were observed during elevation to obtain a functional diagnosis for individual guidance of treatment. ${ }^{524}$ The principal focus was on relearning of normal movement patterns, which could then be transferred to daily activities. ${ }^{5}$ The initial aim was to unload the stress on the rotator cuff and subacromial structures. ${ }^{5}$ During this phase, a mirror for awareness of posture, manual techniques for loosening tense muscles, an elastic rubber band, and a sling fixed to the ceiling were used..$^{524}$ The patients received immediate feedback and correction (supervision) by the physiotherapist. 
Table 1|Baseline characteristics of participant according to treatment group. Values are numbers (percentages) unless stated otherwise

\begin{tabular}{lcc} 
Characteristics & $\begin{array}{c}\text { Supervised exercises } \\
(\mathrm{n}=52)\end{array}$ & $\begin{array}{c}\text { Radial extracorporeal } \\
\text { shockwave treatment }(\mathrm{n}=52)\end{array}$ \\
Mean (SD) age (years) & $49(9.3)$ & $47(11.7)$ \\
\hline Women/men & $26 / 26$ & $26 / 26$ \\
\hline Dominant arm affected & $33(63)$ & $35(67)$ \\
\hline Education: & $30(58)$ & $29(56)$ \\
\hline$\leq 12$ years at school & $22(42)$ & $22(42)$ \\
\hline University/college & $19(37)$ & $15(29)$ \\
\hline Duration of symptoms: & $15(29)$ & $6(12)$ \\
\hline 3-6 months & $8(15)$ & $16(31)$ \\
\hline 6-12 months & $10(19)$ & $24(46)$ \\
\hline $12-24$ months & & $20(38)$ \\
\hline >24 months & $23(44)$ & $62.9(20.1)$ \\
\hline Previous treatments: & $27(52)$ & $1.5(0.5)$ \\
\hline Physiotherapy & $72.4(15.2)$ & $0.74(0.58-0.76)$ \\
\hline Corticosteroid injection & $0.70(0.53-0.76)$ & \\
\hline Mean (SD) EQ VAS* & $1.5(0.4)$ & \\
\hline Median (interquartile range) EQ5D index† & & \\
\hline Mean (SD) emotional distress (1-4) & & \\
\hline VAS & & \\
\hline
\end{tabular}

$\mathrm{VAS}=$ visual analogue scale.

${ }^{*} 0=$ worst imaginable health state; $100=$ best imaginable health state.

†Evaluates health status: five dimensions with three categories in each.

Once dysfunctional neuromuscular patterns were normalised, endurance exercises were performed with gradually increasing resistance. Principles of closed and open kinetic chain and plyometric exercises were incorporated in the next phase of training. ${ }^{524}$ Patients had an adjusted programme at home, which consisted of correction of alignment during daily living and simple low loaded exercises with a thin elastic cord to provide assistance and resistance to the movement. Simple advice was given.

Radial extracorporeal shockwave treatment (Swiss Dolor Clast, EMS) was provided by a physiotherapist experienced in its use. The treatment was administered once a week for four to six weeks; three to five tender points were treated each time. Points were identified through a patient oriented biofeedback process (insertion of supraspinatus tendon, dorsolaterally below the acromion, and a maximum of three trigger points in the rotator cuff muscles). ${ }^{25}$ The frequency applied was 12-8 Hertz with from 2000 pulses per session, with a pressure between 2.5 and 4.0 Bar, depending on what the patient tolerated without local anaesthetic.

Radial extracorporeal shockwave treatment uses low to medium energy shockwaves generated when a projectile is accelerated by compressed air and hits an applicator. $^{7}$ These impulses are delivered into the tissue and spread as spherical "radial" waves (rather than being focused). Patients were informed that the suggested mechanism for pain relief was hyperstimulation analgesia and increased neovascularisation that improves regeneration of tissue. ${ }^{625}$ Patients were advised to avoid activities that elicited pain.

All the patients were asked not to have any additional treatment except analgesics (including antiinflammatory drugs) for their shoulder pain for the time between the start of treatment and the 18 week follow-up.

\section{Sample size}

The study was designed to detect a difference of 10 points in the shoulder pain and disability index score between groups with $\alpha$ value set at 0.05 (type I error) and $\beta$ at 0.2 (type II error). ${ }^{26}$ After a pilot study, and in accordance with a previous study, the standard deviation was estimated to $20 .{ }^{26}$ The sample size was calculated as 48 in each group for a univariate analysis of covariance. ${ }^{27}$ This is a linear model using baseline measures of the primary outcome as a covariate. The $\rho$ was set to $0.5 .^{27}$ The study has three post-treatment registrations.

\section{Statistical analyses}

The main outcome variable (shoulder pain and disability index score) is considered to be a continuous variable. ${ }^{26}$ To evaluate the treatment effect (the mean difference between the groups at six, 12, and 18 weeks), we used the mixed model analysis (repeated measurements). ${ }^{28}$ This model includes the interaction between treatment and elapsed time, baseline values are adjusted, and we assume that data are missing at random. ${ }^{28}$ The distribution of future values for a participant who drops out at time $t$ and one who remains in at time $t$ is expected to be the same if they have the same covariates and the same history of outcome until time t. ${ }^{28}$ Model assumptions were checked by residual plots. We estimated the smallest detectable real difference between two measurements on the same person to be 19.6 points on the shoulder pain and disability index and used this as a cut-off point. ${ }^{19}$ We calculated the number needed to treat (NNT) according to the method of Guyatt et al. ${ }^{29}$

We checked the secondary outcome measures for normal distribution and found that parametric statistics were appropriate. ${ }^{30} \mathrm{We}$ used logistic regression with adjustment for baseline values to compare work status and drug treatment. We analysed data according to the intention to treat principle, in which the study groups are compared in terms of the treatment to which they were randomly allocated.

\section{RESULTS}

A total of 141 patients were eligible for inclusion, and 104 were randomised for study intervention (fig 1). The groups were similar at baseline with regard to age, education, dominant arm affected, duration of pain, sick leave, shoulder pain and disability index score, and secondary outcome variables (tables 1, 2 and 3). Seventeen (33\%) patients in the radial extracorporeal shockwave group and $12(23 \%)$ in the supervised exercise group were on sick leave because of shoulder pain. Five patients in the radial extracorporeal shockwave group and two in the supervised exercise group claimed for disability pension.

Patients treated with radial extracorporeal shockwaves $(n=52)$ received a median of five (interquartile range 4-6) treatments. One patient had only one treatment, and one patient had two treatments. Patients in 
Table 2 Mean (SD) scores and differences in improvement with overall $P$ values at 18 weeks, from mixed models linear (repeated measures analysis)

\begin{tabular}{lcccc}
\multicolumn{1}{c}{$\begin{array}{c}\text { Supervised } \\
\text { exercises }\end{array}$} & $\begin{array}{c}\text { Radial extracorporeal } \\
\text { shockwave treatment }\end{array}$ & $\begin{array}{c}\text { Treatment effect } \\
(95 \% \mathrm{Cl})\end{array}$ & P value \\
Shoulder pain and disability index & & & & \\
\hline Baseline & $48.8(20.6)$ & $45.1(22.1)$ & $-10(-17.6$ to -2.3$)$ & \\
\hline 6 weeks & $25.8(21.5)$ & $33.5(23.3)$ & $-10.3(-19.8$ to -0.8$)$ & \\
\hline 12 weeks & $27(24.2)$ & $36.1(28.4)$ & $-8.4(-16.5$ to -0.6$)$ & 0.047 \\
\hline 18 weeks & $24.5(25.6)$ & $29.2(25.9)$ & &
\end{tabular}

\begin{tabular}{|c|c|c|c|c|}
\hline \multicolumn{5}{|l|}{ At rest: } \\
\hline Baseline & $3.4(1.9)$ & $3.5(2.1)$ & & \\
\hline 6 weeks & $2.6(1.9)$ & $2.9(2.1)$ & $-0.3(-0.9$ to 0.4$)$ & \\
\hline 12 weeks & $2.5(1.8)$ & $2.9(2.1)$ & $-0.3(-0.9$ to 0.3$)$ & \\
\hline 18 weeks & $2.5(1.9)$ & $2.7(2.0)$ & $-0.2(-0.7$ to 0.3$)$ & 0.83 \\
\hline \multicolumn{5}{|c|}{ During activity: } \\
\hline Baseline & $5.6(2.0)$ & $5.4(1.9)$ & & \\
\hline 6 weeks & $3.9(2.0)$ & $4.6(2.4)$ & $-0.7(-1.6$ to 0.1$)$ & \\
\hline 12 weeks & $3.7(2.2)$ & $4.1(2.4)$ & $-0.5(-1.3$ to 0.4$)$ & \\
\hline 18 weeks & $3.6(2.3)$ & $4.1(2.5)$ & $-0.6(-1.3$ to 0.2$)$ & 0.42 \\
\hline
\end{tabular}

\section{Function}

Carrying bag:

\begin{tabular}{lllll}
\hline Baseline & $4.1(1.8)$ & $3.6(2.0)$ & & \\
\hline 6 weeks & $3.0(1.8)$ & $3.1(2.1)$ & $-0.5(-1.1$ to 0.2$)$ & \\
\hline 12 weeks & $3.0(1.9)$ & $3.2(2 . .0)$ & $-0.4(-1.0$ to 0.2$)$ & \\
\hline 18 weeks & $2.8(1.8)$ & $3.0(2.1)$ & $-0.5(-1.0$ to 0.1$)$ & 0.26 \\
\hline
\end{tabular}

Taking down from

cupboard:

\begin{tabular}{lllll}
\hline Baseline & $4.9(1.2)$ & $4.6(1.8)$ & & \\
\hline 6 weeks & $3.4(1.7)$ & $3.9(1.9)$ & $-0.7(-1.3$ to -0.01$)$ & \\
\hline 12 weeks & $3.1(1.9)$ & $3.5(2.0)$ & $-0.5(-1.2$ to 0.2$)$ & \\
\hline 18 weeks & $3.2(1.8)$ & $3.4(2.0)$ & $-0.5(-1.1$ to 0.1$)$ & 0.2 \\
\hline
\end{tabular}

the supervised exercise group $(\mathrm{n}=51)$ received a median of 14 (11-16) treatments. One patient did not receive supervised exercises because of depression, and another had only four sessions because of an increase in pain and a suspected adhesive capsulitis. One patient crossed over to the supervised exercise group after one treatment with radial extracorporeal shockwaves (fig 1). Fourteen patients did not return the questionnaire at six weeks. Two patients randomised to supervised exercises did not attend followup at 12 and 18 weeks, and two patients in the radial extracorporeal shockwave group did not attend the 18 week follow-up (fig 1 ). Thirteen patients in the radial extracorporeal shockwave group and three patients in the supervised exercise group received additional treatment (cortisone injections, chiropractic treatment, physical therapy/supervised exercises) between 12 and 18 weeks (odds ratio 5.5, 95\% confidence interval 1.3 to $26.4 ; \mathrm{P}=0.014$ ).

\section{Primary outcome}

The treatment effect was in favour of supervised exercises at six, 12, and 18 weeks. At 18 weeks the treatment effect was -8.4 (95\% confidence interval -16.5 to -0.6 ; $\mathrm{P}=0.047$ ) points (table 2, fig 2). The treatment effect was consistent when adjusted for $\operatorname{sex}(\mathrm{P}=0.049)$. Thirty two out of $50(64 \%)$ patients treated with supervised exercises and $18 / 50(36 \%)$ patients treated with radial extracorporeal shockwaves achieved a reduction in shoulder pain and disability index score exceeding the smallest detectable difference of 19.6 points (odds ratio 3.2, 1.3 to $7.8 ; \mathrm{P}=0.009$ ). Two (4\%) patients in the group of supervised exercises and three $(6 \%)$ in the radial extracorporeal shockwave group deteriorated after treatment. The number needed to treat was 3.2 (95\% confidence interval 2.1 to 7.1 ).

\section{Secondary outcomes}

At 18 weeks, results for pain, function, and active range of motion were not statistically significant (table 2). More patients in the supervised exercise group returned to work $(\mathrm{P}=0.016)$ (table 3$)$. Six patients in the supervised exercise group and one in the radial extracorporeal shockwave group used less drug treatment (table 3).

\section{Adverse events}

One patient in the supervised exercise group reported a considerable increase in pain and stiffness consistent with adhesive capsulitis and had only four treatments. In the group treated with radial extracorporeal shockwaves, one patient dropped out after one treatment and one after two treatments because of the aggravation of pain; one of them crossed over to the supervised exercise group (table 4).

\section{DISCUSSION}

We found a small but statistically significant difference in favour of supervised exercises over radial extracorporeal shockwave treatment for the primary outcome (shoulder pain and disability index) at six, 12, and 18 weeks in patients with subacromial shoulder pain. The difference was consistent after adjustment for education and duration of pain. In addition, more patients from the radial extracorporeal shockwave group (13 $v 03$ ) had additional treatment after 12 weeks, suggesting that they were less satisfied. The results for the differences in secondary outcomes were in favour of exercises, but differences were not

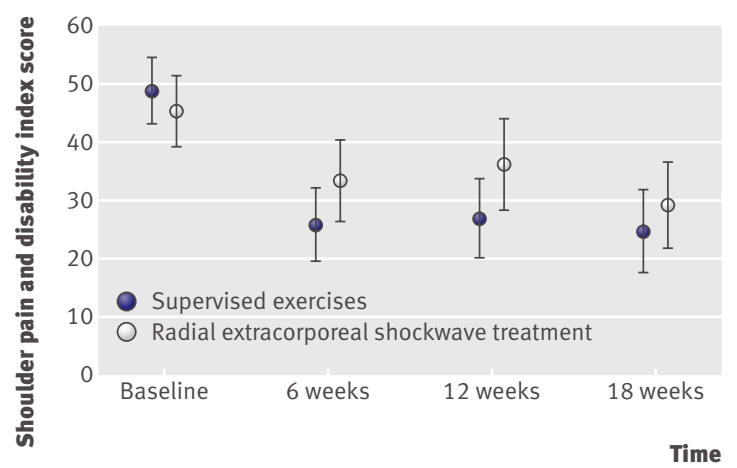

Fig 2 | Mean shoulder pain and disability index scores with $95 \%$ confidence intervals for supervised exercises and radial extracorporeal shockwave treatment 
Table $3 \mid$ Work status and drug treatment. Values are numbers (percentages) unless stated otherwise

\begin{tabular}{lccc}
$\begin{array}{l}\text { Outcome } \\
\text { Working*: }\end{array}$ & $\begin{array}{c}\text { Supervised } \\
\text { exercises }\end{array}$ & $\begin{array}{c}\text { Radial extracorporeal } \\
\text { shockwave treatment }\end{array}$ & P value \\
\hline Baseline & $31 / 52(60)$ & $26 / 52(50)$ & 0.6 \\
\hline 12 weeks & $32 / 50(64)$ & $28 / 52(54)$ & 0.016 \\
\hline 18 weeks & $38 / 50(76)$ & $26 / 50(52)$ & \\
\hline Drug treatment† (daily/each week): & & $23 / 52(44)$ & 0.26 \\
\hline Baseline & $26 / 52(50)$ & $22 / 50(44)$ & \\
\hline 18 weeks & $18 / 50(36)$ & & \\
\hline
\end{tabular}

* Old age pension not included (four in supervised exercise group and two in radial extracorporeal shockwave group at all follow-ups).

†Includes drug treatments for pain, sleep problems, and depression.

significant except for change in work status after 18 weeks, which may indicate that supervised exercise is a more comprehensive rehabilitation intervention.

\section{Strengths and limitations}

The advantages of this study are the randomised design, stratification by sex, treatments provided by physiotherapists experienced in the use of the methods, high compliance, blinded observer, large number of patients attending follow-up, and intention to treat analysis.

At least three possible limitations must be taken into account when interpreting the results. Firstly, we did not include a placebo group and cannot exclude the possibility that the observed results reflect a placebo effect or the natural course of the condition. A previous trial reported that a supervised exercise regimen was superior to placebo, so we found it difficult to justify the inclusion of a placebo group. ${ }^{21}$ On the other hand, two trials found no difference between sham and extracorporeal shockwave treatment. ${ }^{714} \mathrm{We}$ did not include a placebo radial extracorporeal shockwave group and cannot substantiate the placebo effect in our study. Secondly, local anaesthetics were not injected into the subacromial space to improve diagnostic accuracy. On the other hand, rotator cuff disease or subacromial shoulder pain is a clinical diagnosis and we used the recommended combination of clinical tests to improve patient selection. ${ }^{17}$ Thirdly, the study may be underpowered for detecting differences in the secondary outcome variables. We used a univariate analysis of covariance to calculate the sample size with regard to the primary outcome. ${ }^{27}$

Table $4 \mid$ Patients who changed treatment or diagnosis within first 18 weeks

\begin{tabular}{|c|c|c|}
\hline Reason & $\begin{array}{l}\text { Supervised } \\
\text { exercises }\end{array}$ & $\begin{array}{l}\text { Radial extracorporeal } \\
\text { shockwave treatment }\end{array}$ \\
\hline Adhesive capsulitis & 2 & 1 \\
\hline Polymyalgia rheumatica & & 1 \\
\hline Depression & 1 & \\
\hline Crossed over to supervised exercises before 18 weeks & 0 & $2^{\star}$ \\
\hline Operated on before 18 weeks & 0 & 1 \\
\hline
\end{tabular}

\section{WHAT IS ALREADY KNOWN ON THIS TOPIC}

Supervised exercises and arthroscopic surgery are better than placebo treatment for shoulder pain

Moderate evidence suggests that low energy radial extracorporeal shockwave treatment is not effective for noncalcifying rotator cuff tendinosis

\section{WHAT THIS STUDY ADDS}

Supervised exercises are better than radial extracorporeal shockwave treatment for short term improvement in patients with subacromial shoulder pain

More patients treated with supervised exercises returned to work

\section{Comparison with existing literature}

Our results are in agreement with results from previous trials recommending exercise therapy ${ }^{9-1131}$ and do not strengthen the evidence for extracorporeal shockwave treatment. ${ }^{67141532}$ The difference in shoulder pain and disability index score of 8.4 points at the 18 week follow-up was somewhat smaller than the difference (10 points) the study was designed to detect. For interpreting the effectiveness of the treatment, we must consider not only statistical significance but also whether the improvement (or deterioration) is relevant for the patients or clinicians. ${ }^{33}$ By using the smallest detectable real difference for an individual patient of 19.6 points as a cut-off point, we found that a larger proportion of patients in the supervised exercise group than in the radial extracorporeal shockwave treatment group improved. ${ }^{19}$ This suggests that more patients receiving supervised exercises had clinically relevant improvement. ${ }^{1934}$ The number needed to treat to benefit from supervised exercises was three, which is considered clinically important. ${ }^{29}$ However, the estimate of number needed to treat has to be interpreted with caution because of possible differences in the study population recruited from a university hospital and the population in primary care to which we wish to extrapolate.

The shoulder pain and disability index is a combined score reported to be one of the most responsive shoulder specific questionnaires available and more responsive than single subjective evaluations of pain and function or objective measures of range of motion. ${ }^{19}$ The observed improvements were largest at six weeks, suggesting that a reduction of mechanical subacromial stress and normalisation of movement patterns had occurred within a relatively short treatment period. The content of the supervised exercise intervention was slightly different from the study of Brox et al. ${ }^{21}$ Fewer patients used a sling for home exercises in our study; all supervision was finished within 12 weeks; and separate lessons on functional anatomy, pain management, and ergonomics were not included. Follow-up in Brox et al's study was at three and six months and two years. 


\section{Conclusions}

After 18 weeks, supervised exercises were better than radial extracorporeal shockwave treatment in terms of the primary outcome variable - the shoulder pain and disability index - and one secondary outcome variable -work status. We found no significant differences for the other secondary outcome variables of pain, function, active range of motion, and use of drug treatment. More patients in the supervised exercise group improved, probably owing to a treatment effect.

We thank Stine 0 Eriksen for doing all the assessments, Roald Danielsen for administering radial extracorporeal shockwave treatment, and Mona Mortensen and Helene Skaara for leading the supervised exercises. We also thank Egil Knag, Enimed, for lending us the Swiss Dolor Clast. A special thanks to all the patients who made this study possible.

Contributors: KE contributed to the study design; data collection, analysis, and interpretation; and writing of the manuscript. MG and JIB contributed to the study design, data analysis and interpretation, and writing of the manuscript. EB-H contributed to the study design and data collection and interpretation. LS contributed to data analysis and interpretation. NG) and OME contributed to the study design and data collection and interpretation. $\mathrm{KE}$ is the guarantor.

Funding: The study was supported by Health Region East, Norway. Competing interests: None declared.

Ethical approval: The study protocol was approved by the ethics committee for Medical Research Region 1 of Norway.

1 Faber E, Kuiper JI, Burdorf A, Miedema HS, Verhaar JA. Treatment of impingement syndrome: a systematic review of the effects on functional limitations and return to work. J Occup Rehabil 2006;16:7-25

2 Brox Jl. Shoulder pain. Best Pract Res Clin Rheumatol 2003;17:33-56

3 Blaine TA, Kim YS, Voloshin I, Chen D, Murakami K, Chang SS, et al. The molecular pathophysiology of subacromial bursitis in rotator cuff disease. J Shoulder Elbow Surg 2005;14(1 suppl S):84-9S.

4 Green S, Buchbinder R, Glazier R, Forbes A. Systematic review of randomised controlled trials of interventions for painful shoulder: selection criteria, outcome assessment, and efficacy. BMJ 1998;316:354-60.

5 Bohmer A, Staff PH, Brox II. Supervised exercises in relation to rotato cuff disease (impingement syndrome, stages 1 and II): a treatment regimen and its rationale. Physiother Theory Pract 1998;14:93-105.

6 Harniman E, Carette S, Kennedy C, Beaton D. Extracorporeal shock wave therapy for calcific and noncalcific tendonitis of the rotator cuff: a systematic review. J Hand Ther 2004;17:132-51.

7 Cacchio A, Paoloni M, Barile A, Don R, de Paulis F, Calvisi V, et al. Effectiveness of radial shock-wave therapy for calcific tendinitis of the shoulder: single-blind, randomized clinical study. Phys Ther 2006;86:672-82.

8 Buchbinder R, Green S, Youd JM. Corticosteroid injections for shoulder pain. Cochrane Database Syst Rev 2003;(1):CD004016.

9 Hay EM, Thomas E, Paterson SM, Dziedzic K, Croft PR. A pragmatic randomised controlled trial of local corticosteroid injection and physiotherapy for the treatment of new episodes of unilateral shoulder pain in primary care. Ann Rheum Dis 2003;62:394-9.

10 Lombardi I Jr, Magri AG, Fleury AM, Da Silva AC, Natour J. Progressive resistance training in patients with shoulder impingement syndrome: a randomized controlled trial. Arthritis Rheum 2008;59:615-22.

11 Coghlan JA, Buchbinder R, Green S, Johnston RV, Bell SN. Surgery for rotator cuff disease. Cochrane Database Syst Rev 2008;(1):CD005619.

12 Unruh AM. Gender variations in clinical pain experience. Pain 1996;65:123-67.
13 Zheng Z, Simpson JA, van der Windt D, Elliott AM. Data from a study of effectiveness suggested potential prognostic factors related to the patterns of shoulder pain. J Clin Epidemiol 2005;58:823-30.

14 Speed CA, Richards C, Nichols D, Burnet S, Wies JT, Humphreys H, et al. Extracorporeal shock-wave therapy for tendinitis of the rotator cuff. J Bone Joint Surg Br 2002;84:509-12.

15 Schmitt J, Haake M, Tosch A, Hildebrand R, Deike B, Griss P. Lowenergy extracorporeal shock-wave treatment (ESWT) for tendinitis of the supraspinatus: a prospective, randomised study. J Bone Joint Surg Br 2001;83:873-6.

16 Michener LA, Walsworth MK, Burnet EN. Effectiveness of rehabilitation for patients with subacromial impingement syndrome. Hand Ther 2004;17:152-64.

17 Park HB, Yokota A, Gill HS, El Rassi G, McFarland EG. Diagnostic accuracy of clinical tests for the different degrees of subacromial impingement syndrome. J Bone Joint Surg Am 2005;87:1446-55.

18 Roach KE, Budiman-Mak E, Songsiridej N, Lertratanakul Y. Development of a shoulder pain and disability index. Arthritis Care Res 1991;4:143-9.

19 Ekeberg OM, Bautz-Holter E, Tveita EK, Keller A, Juel NG, Brox JI. Agreement, reliability and validity in 3 shoulder questionnaires in patients with rotator cuff disease. BMC Musculoskelet Disord 2008;9:68.

20 Tveita EK, Tariq R, Sesseng S, Juel NG, Bautz-Holter E. Hydrodilatation, corticosteroids and adhesive capsulitis: a randomized controlled trial. BMC Musculoskelet Disord 2008;9:53.

21 Brox JI, Staff PH, Ljunggren AE, Brevik JI. Arthroscopic surgery compared with supervised exercises in patients with rotator cuff disease (stage II impingement syndrome). BMJ 1993;307:899-903.

22 Triffitt PD, Wildin C, Hajioff D. The reproducibility of measurement of shoulder movement. Acta Orthop Scand 1999;70:322-4.

23 Holm I, Friis A, Storheim K, Brox JI. Measuring self-reported functional status and pain in patients with chronic low back pain by postal questionnaires: a reliability study. Spine 2003;28:828-33.

24 Virta L, Mortensen M, Eriksson R, Âller M. How many patients with subacromial impingement syndrome recover with physiotherapy? A follow-up study of a supervised exercise programme. Adv Physiother 2008;11:1-8.

25 Gerdesmeyer L, Gollwitzer H, Diehl P, Wagner K. Radial extracorporeal shockwave therapy (rESWT) in orthopaedics. J Miner Stoffwechs 2004;11:36-9.

26 Williams JW, Hollemann DR, Simmel DL. Measuring shoulder function with the shoulder pain and disability index. J Rheumatol 1995;22:727-32.

27 Frison L, Pocock SJ. Repeated measures in clinical trials: analysis using mean summary statistics and its implications for design. Stat Med 1992;11:1685-704

28 Fitzmaurice GM. Applied longitudinal analysis. Hoboken, NJ: WileyInterscience, 2004

29 Guyatt GH, Juniper EF, Walter SD, Griffith LE, Goldstein RS. Interpreting treatment effects in randomised trials. $B M J$ 1998;316:690-3.

30 Vickers AJ. Parametric versus non-parametric statistics in the analysis of randomized trials with non-normally distributed data. BMC Med Res Methodol 2005;5:35.

31 Green S, Buchbinder R, Hetrick S. Physiotherapy interventions fo shoulder pain. Cochrane Database Syst Rev 2003;(2):CD004258.

32 Gerdesmeyer L, Wagenpfeil S, Haake M, Maier M, Loew M, Wortler K, et al. Extracorporeal shock wave therapy for the treatment of chronic calcifying tendonitis of the rotator cuff: a randomized controlled trial. JAMA 2003;290:2573-80.

33 De Vet HC, Terwee CB, Knol DL, Bouter LM. When to use agreement versus reliability measures. J Clin Epidemiol 2006;59:1033-9.

34 De Vet HC, Ostelo R, Terwee C, van der Roer N, Knol D, Beckerman H, et al. Minimally important change determined by a visual method integrating an anchor-based and a distribution-based approach. Qual Life Res 2007;16:131-42.

Accepted: 25 May 2009 